\title{
On signed incomplete Cholesky factorization preconditioners for saddle-point systems
}

\section{JA Scott, M Tuma}

February 2014 
RAL Library

STFC Rutherford Appleton Laboratory

R61

Harwell Oxford

Didcot

OX11 0QX

Tel: $+44(0) 1235445384$

Fax: $+44(0) 1235446403$

email: libraryral@stfc.ac.uk

Science and Technology Facilities Council preprints are available online at: http:// epubs.stfc.ac.uk

ISSN 1361- 4762

Neither the Council nor the Laboratory accept any responsibility for loss or damage arising from the use of information contained in any of their reports or in any communication about their tests or investigations. 


\title{
On signed incomplete Cholesky factorization preconditioners for saddle-point systems
}

\author{
Jennifer Scott $^{1}$ and Miroslav Tůma ${ }^{2}$
}

\begin{abstract}
Limited-memory incomplete Cholesky factorizations can provide robust preconditioners for sparse symmetric positive-definite linear systems. In this paper, the focus is on extending the approach to sparse symmetric indefinite systems in saddle-point form. A limited-memory signed incomplete Cholesky factorization of the form $L D L^{T}$ is proposed, where the diagonal matrix $D$ has entries \pm 1 . The main advantage of this approach is its simplicity as it avoids the use of numerical pivoting. Instead, a global shift strategy involving two shifts (one for the $(1,1)$ block and one for the $(2,2)$ block of the saddle-point matrix) is used to prevent breakdown and to improve performance. The matrix is optionally prescaled and preordered using a standard sparse matrix ordering scheme that is then post-processed to give a constrained ordering that reduces the likelihood of breakdown and need for shifts. The use of intermediate memory (memory used in the construction of the incomplete factorization but subsequently discarded) is shown to significantly improve the performance of the resulting preconditioner. Some new theoretical results are presented and for problems arising from a range of practical applications, numerical results are given to illustrate the effectiveness of the signed incomplete Cholesky factorization as a preconditioner. Comparisons are made with a recent incomplete $L D L^{T}$ code that employs pivoting.
\end{abstract}

Keywords: sparse matrices, sparse linear systems, indefinite symmetric systems, saddle-point systems, iterative solvers, preconditioning, incomplete Cholesky factorization.

AMS(MOS) subject classifications: 65F05, 65F50

1 Scientific Computing Department, Rutherford Appleton Laboratory, Harwell Oxford, Oxfordshire, OX11 0QX, UK.

Correspondence to: jennifer.scott@stfc.ac.uk

Supported by EPSRC grant EP/I013067/1.

2 Institute of Computer Science, Academy of Sciences of the Czech Republic.

Partially supported by the Grant Agency of the Czech Republic Project No. P201/13-06684 S.

Travel support from the Academy of Sciences of the Czech Republic is also acknowledged.

February 6, 2014 


\section{Introduction}

We are interested in solving linear systems $K x=b$ where the sparse symmetric matrix $K$ is of the form

$$
K=\left(\begin{array}{cc}
A & B^{T} \\
B & -C
\end{array}\right)
$$

Here $A$ is $n \times n$ symmetric positive definite, $B$ is rectangular $m \times n$ and of full $\operatorname{rank}(m \leq n)$, and $C$ is $m \times m$ symmetric positive semi-definite. Matrices of the form (1.1) are often called saddle-point matrices or, in the special case $C=0$, KKT matrices, in reference to the Karush-Kuhn-Tucker first-order necessary optimality conditions for the solution of general nonlinear programming problems. Saddle-point problems arise frequently in many applications in science and engineering, including in equality and inequality constrained nonlinear programming, interior point algorithms in both linear and nonlinear optimization, sparse optimal control, mixed finite-element discretizations of partial differential equations in fields such as fluid and solid mechanics, and circuit analysis.

Typically, $K$ is large. In some cases it may be possible as well as desirable to use a direct solver; indeed, a number of sparse direct solvers (including the packages MA57 [16] and HSL_MA86 [31] from the HSL mathematical software library [34]) have been designed with such systems in mind. However, the memory demands of direct solvers can mean that, for very large $K$, it is necessary to use an iterative method, usually a Krylov subspace-based method (see, for example, [62]). Moreover, in some applications it may not be necessary to solve the system with high accuracy or the linear solve may be part of a nonlinear iteration and here an iterative method may then be the method of choice. Unfortunately, Krylov methods tend to converge very slowly when applied to saddle-point systems and a good preconditioner is needed to accelerate convergence. Over the last 20 years or so, a vast amount of work has been devoted to the development of effective preconditioners for saddle-point problems. A quick search of the literature reveals numerous publications incorporating a wide range of application areas; an excellent survey of the work done up to 2005, together with a comprehensive reference list, is given in Section 10 of [4] (see also [5] for a later and more concise overview).

As discussed in $[4,5]$, much effort has focused on the development of block diagonal and block triangular preconditioners as well as on constraint preconditioners. Comparatively little work appears to have concentrated on the development of reliable incomplete factorization techniques for saddle-point systems. Following work done on generating good orderings and scalings for direct solvers for indefinite systems [19, 53], Hagemann and Schenk [29] proposed using a maximum weighted matching algorithm to preprocess the matrix. The objective is to permute large entries on to the subdiagonal that can then be used to form stable $2 \times 2$ pivots, allowing the incomplete factorization to avoid the use of dynamic pivoting (which can add a computational overhead as well as significantly complicating the software development). If a pivot is found to be too small, breakdown is prevented by adding a small perturbation. In [29], some good results are reported (sparse factors and lower iteration counts) for tests on saddle-point problems.

$\mathrm{Li}$ and Saad [37] developed pivoting strategies for sparse symmetric matrices to improve the robustness of their Crout variant of $I L U$ preconditioner [38]. They found that incorporating Bunch-Kaufman pivoting [10] can be efficiently and effectively integrated within an incomplete factorization for sparse symmetric indefinite matrices and reported some encouraging results for general indefinite problems as well as for some KKT problems. Using the work of Li and Saad, Greif, He and Liu [28] have recently made available an incomplete factorization package called SYM-ILDL that is designed for general symmetric indefinite matrices. This package optionally starts by scaling the matrix to be equilibrated in the maximum norm, and then preorders using the Reverse Cuthill-McKee algorithm or approximate minimum degree. As in $\mathrm{Li}$ and Saad, there is an option to use Bunch-Kaufman pivoting during the factorization to maintain stability and avoid breakdown. The user controls the maximum allowed fill within each column and also the dropping of small entries. Currently, no published results are available for this new code but initial findings appear consistent with those reported by Li and Saad [27] (see also Section 6 below).

For saddle-point systems, an alternative approach is based on the observation that $K$ can be factored 
into the form

$$
K=\left(\begin{array}{cc}
A & B^{T} \\
B & -C
\end{array}\right)=\left(\begin{array}{cc}
L_{11} & 0 \\
L_{21} & L_{22}
\end{array}\right)\left(\begin{array}{cc}
I & 0 \\
0 & -I
\end{array}\right)\left(\begin{array}{cc}
L_{11}^{T} & L_{21}^{T} \\
0 & L_{22}^{T}
\end{array}\right)
$$

where

$$
A=L_{11} L_{11}^{T}
$$

is the Cholesky factorization of $A$,

$$
L_{21}=B L_{11}^{-T}
$$

and

$$
S=C+L_{21} L_{21}^{T}=L_{22} L_{22}^{T}
$$

is the Cholesky factorization of the (negative) Schur complement [48, 67]. Note that this factorization always exists, without pivoting (although numerical stability is not guaranteed). Bridson [9] refers to (1.2) as a signed Cholesky factorization. If we set

$$
\mathcal{L}=\left(\begin{array}{cc}
L_{11} & 0 \\
L_{21} & L_{22}
\end{array}\right)
$$

then $P=\mathcal{L L}^{T}$ can be used as a (split) preconditioner. Since the preconditioned matrix $\mathcal{L}^{-1} K \mathcal{L}^{-T}$ has two distinct eigenvalues \pm 1 , a symmetric Krylov subspace method such as MINRES or SYMMLQ [46] would converge in at most two iterations. In practice, the exact factor $\mathcal{L}$ is replaced by an incomplete one. This can be achieved by first computing an incomplete Cholesky $(I C)$ factorization

$$
A \approx \tilde{L}_{11} \tilde{L}_{11}^{T}
$$

which can be done using one of the many available approaches (see, for example, [57] for an historical overview and a list of references to work done since the 1950s). The second step is to compute a sparse approximation $\tilde{L}_{21}$ to $L_{21}$ by solving the upper triangular system

$$
\tilde{L}_{11}^{T} \tilde{L}_{21}=B^{T}
$$

and then possibly applying some dropping criteria to preserve sparsity in $\tilde{L}_{21}$. Finally, an $I C$ factorization of the approximate Schur complement

$$
C+\tilde{L}_{21} \tilde{L}_{21}^{T} \approx \tilde{L}_{22} \tilde{L}_{22}^{T}
$$

is computed. The resulting incomplete factor

$$
\tilde{\mathcal{L}}=\left(\begin{array}{cc}
\tilde{L}_{11} & 0 \\
\tilde{L}_{21} & \tilde{L}_{22}
\end{array}\right)
$$

and its transpose can then be used to define a positive-definite factorized preconditioner $\tilde{P}=\tilde{\mathcal{L}} \tilde{\mathcal{L}}^{T}$. Some very limited numerical results using the SYMMLQ and Uzawa [22] algorithms are given in [48]; a more comprehensive study of how this approach performs in practice (and, in particular, how to obtain a good sparse approximation $\tilde{L}_{21}$ to $L_{21}$ ) appears to be currently lacking. This is something we plan to report on in the future, using our incomplete Cholesky factorization code HSL_MI28 [56] to compute each of the two $I C$ factorizations.

In this paper, we take a different approach to obtain an incomplete factorization. Our idea is to compute a signed $I C$ factorization. The signed complete Cholesky factorization approach has been used for saddle-point systems by, for example, Bridson [9] (and see also [54]). A signed $I C$ approach is attractive for us as it allows us to use a modified version of our existing $I C$ factorization code and, at the same time, permits the exploitation of more general orderings that are not restricted to ordering the $(1,1)$ block of the matrix separately from the $(2,2)$ block. Allowing the use of more general permutations appears to be a 
preferable strategy when factorizing KKT systems, as discussed and demonstrated, for example, by Fourer and Mehrotra in their paper [23] on solving the indefinite linear systems that arise in an interior-point method. Note that the signed factorization avoids the need to compute an explicit sparse approximation of $\tilde{L}_{21}$ as this block is part of the global Cholesky-like factor. Importantly, we also avoid the need for pivoting and the use of $2 \times 2$ pivots (but see the comments on the stability of this approach below). Performing threshold partial pivoting at each step of the factorization process not only complicates the implementation but can add a significant time overhead. See also the related motivation for static pivoting introduced for $L U$ factorizations in [39].

Before describing our approach in more detail, we briefly mention similar concepts for obtaining an unreduced Cholesky-like factorization, that is, the factorization algorithm is applied to the whole matrix $K$, not preceded by the null-space or range-space transformations of the matrix blocks used in segregated approaches [4]. If the full rank condition for the $B$ block in (1.1) is replaced by positive definiteness of the $C$ block we get a class of symmetric quasi-definite (SQD) matrices. Vanderbei [63] shows that SQD matrices are strongly factorisable, i.e., a signed Cholesky factorization exists for such matrices under any symmetric permutation; this is a stronger result than we have for our saddle-point matrix $K$. Further, a stability analysis related to the factorization of SQD matrices is given by Gill et al [24] (see also [25]), which shows the importance of the effective condition number of $K$ for the stability of the factorization. In particular, the effective condition number is small if the maximum of the norms $\left\|B^{T} A^{-1} B\right\|$ and $\left\|B^{T} C^{-1} B\right\|$ is small with respect to the norm of $K$. Also note that the analysis of a symmetric indefinite factorization based on the related generalized $Q R$ factorization in [49] points out that conditioning of the principal leading submatrices may be determined by other factors such as the number of sign changes in the diagonal of the signed factorization. In practice, neither the SQD condition or the full rank condition for the block $B$ needs to be satisfied. Instead, the existence of a signed Cholesky factorization can be forced by "regularization", that is, by modifying the diagonal entries. Saunders and Tomlin [52] used fixed regularization parameters (diagonal shifts) for the blocks $A$ and $-C$ to ensure stability a priori. After regularization of both blocks, the signed Cholesky factorization always exists and they used this approach to successfully solve test problems from the Netlib collection (http://www.netlib.org/lp/data/) via the barrier method using a sparse direct Cholesky solver. Dynamic regularization by perturbing diagonal entries in the signed Cholesky factorization that results in a SQD matrix was proposed by Altman and Gondzio [2]. This strategy seems to introduce less perturbation to the logarithmic barrier method used to solve optimization problems: see also numerical comparisons and notes on signed Cholesky and indefinite factorizations in $[1,6,8]$. While Altman and Gondzio use a complete signed Cholesky factorization within the HOPDM optimization code [26], the diagonal entry modification in case of a wrong sign (the modification should enforce positive definiteness of $A$ and negative definiteness of $-C$ ) or an entry of small magnitude is based on that originally proposed by Kershaw [36]. Another regularization strategy combined with reorderings restricted to blocks (priority minimum degree) of the saddle-point problem was proposed by Vanderbei and Shannon [65] and implemented in the optimization code LOQO [64]. They shift the diagonal of the block corresponding to the primal variables by adding a multiple of a unit matrix. Moreover, in order to get a strong (complete) factorization of the regularized matrix, they allow both increases and decreases to the shifts in a manner that is similar to the strategy proposed by Manteuffel [41] and as implemented, for example, by Lin and Moré [40], see also [56]. Thus the signed Cholesky factorization has attracted attention because of its simplicity and because of the systems to be solved, often obtained in sequence, may need regularization. More sophisticated and expensive dynamic reorderings that combine symbolical and numerical phases may be then considered as an overkill. Note that the complete signed Cholesky implementation was also popular in solving indefinite systems within shift-and-invert based eigensolvers, especially in engineering communities. An example is the implementation of SKYPACK [42] for the block Lanczos eigenvalue software BLZPACK (http://crd-legacy.lbl.gov/ osni/\#Software), where part of the motivation to use a signed Cholesky factorization comes from the choice of skyline data structure for the sparse matrices.

Let us summarize the reasons leading us to develop a state-of-the-art approach to solve a large class of 
indefinite systems. Firstly, as already mentioned, we are interested in preconditioned iterative methods in order to be able to solve very large problems. Secondly, we want to exploit orderings that are as general as possible since general orderings are often claimed to lead to the most efficient solvers. Furthermore, an important number of real-world problems need to be regularized and algebraic modifications seem to be very natural. Our previous project $[56,57]$ considered a large spectrum of different application areas leading to linear systems with symmetric positive-definite system matrices and found that regularization by flexible strategies based on the Manteuffel shifts [41] is preferable to ad-hoc modifications. We are keen to use such a strategy in a signed $I C$ code. Lastly, but not least, we want preconditioners based on incomplete factorizations that are not only time and memory efficient but also robust.

The rest of the paper is organised as follows. In Section 2, we briefly recall the signed Cholesky factorization approach using the description given by Bridson. Then in Section 3, we summarize our limited memory $I C$ factorization for positive-definite problems. This is extended to a signed $I C$ factorization in Section 4. In Section 5, some theoretical results for the signed $I C$ approach are given. Numerical results, including comparisons with SYM-ILDL, are presented in Section 6 and concluding remarks are made in Section 7 .

\section{Constrained ordering and signed $I C$ factorizations}

In this section, we consider the case of a complete factorization of the matrix (1.1). The aim of a constrained ordering is to find a permutation $Q$ such that $Q K Q^{T}$ can be factorized stably without the need for numerical pivoting and without modifying the entries in $K$, while still limiting the number of entries in the factor. This problem has been examined for special classes of matrices by a number of authors. Of practical interest is the class of $\mathcal{F}$ matrices, where each column of $B$ has exactly two entries which sum to zero and $C=0$. These arise in, for example, Stokes flow problems. Tưma [60] and De Niet and Wubs [15] present methods for these problems while Bridson [9] proposed a constrained ordering for more general saddle-point problems.

We use the terminology of Bridson and divide the nodes of the adjacency graph of the matrix $K$ into two disjoint sets: those that correspond to the diagonal entries of $A$ are known as $A$-nodes and the remaining nodes as $C$-nodes. The ordering constraint proposed in [9] is extremely simple: a $C$-node can only be ordered after all its $A$-node neighbours in the graph of $K$ have been ordered. Bridson has the following result, which is included here for completeness.

Theorem 2.1. Let $A$ be positive definite, $C$ be positive semi-definite and $B$ be of full row rank. Then if a $C$-node is ordered only after all its A-node neighbours, the signed Cholesky factorization

$$
P K P^{T}=L D L^{T}
$$

exists, where $P$ is a permutation matrix corresponding to the ordering of the nodes, $L$ is a lower triangular matrix with positive diagonal entries and $D$ is a diagonal matrix with entries \pm 1 .

Proof: First observe that the inverse of a saddle-point matrix satisfying the above conditions can be expressed as

$$
\left(\begin{array}{cc}
A & B^{T} \\
B & C
\end{array}\right)^{-1}=\left(\begin{array}{cc}
A^{-1}-A^{-1} B^{T} S^{-1} B A^{-1} & A^{-1} B^{T} S^{-1} \\
S^{-1} B A^{-1} & S^{-1}
\end{array}\right),
$$

where the negative Schur complement $S$ given by

$$
S=C+B A^{-1} B^{T}
$$

is positive definite. 
We now proceed by induction. The first node to be eliminated must be an $A$-node with pivot $d_{1}=$ $a_{11}>0$. Assume $i-1$ steps of the factorization have been performed. Partitioning the first $i-1$ nodes into $A$-nodes and $C$-nodes, the $i$-th principal submatrix of (a permutation of) $K$ is

$$
\left(\begin{array}{cc}
K_{i-1} & k_{i,:}^{T} \\
k_{i,:} & k_{i i}
\end{array}\right)=\left(\begin{array}{ccc}
A_{i-1} & B_{i-1}^{T} & u^{T} \\
B_{i-1} & -C_{i-1} & v^{T} \\
u & v & k_{i i}
\end{array}\right),
$$

where $k_{i, \text { : }}$ denotes the first $i-1$ entries in row $i$. Since $A_{i-1}$ is a principal submatrix of the positive-definite matrix $A$, it must also be positive definite. The ordering constraint implies that all the non zeros in the rows of $B$ corresponding to already ordered $C$-nodes must appear in $B_{i-1}$ (otherwise there would be a $C$-node ordered before $i$ with an $A$-node neighbour ordered at $i$ or later). Since $B$ is assumed to have full row rank, $B_{i-1}$ also has full row rank: $B_{i-1}$ is a subset of the rows of $B$ with possibly some fully zero columns deleted. Thus $K_{i-1}$ satisfies the same conditions as $K$.

Case 1: node $i$ is an $A$-node. It follows from the ordering constraint that $i$ can have no previously ordered $C$-node neighbours and so $v=0$ in equation (2.2). Using the form of the inverse in equation $(2.1)$, the pivot is

$$
\begin{aligned}
d_{i i} & =k_{i i}-\left(\begin{array}{ll}
u & 0
\end{array}\right)\left(\begin{array}{cc}
A_{i-1} & B_{i-1}^{T} \\
B_{i-1} & -C_{i-1}
\end{array}\right)^{-1}\left(\begin{array}{c}
u^{T} \\
0
\end{array}\right) \\
& =k_{i i}-u\left(A_{i-1}^{-1}-A_{i-1}^{-1} B_{i-1}^{T} S_{i-1}^{-1} B_{i-1} A_{i-1}^{-1}\right) u^{T} \\
& =\left(k_{i i}-u A_{i-1}^{-1} u^{T}\right)+u A_{i-1}^{-1} B_{i-1}^{T} S_{i-1}^{-1} B_{i-1} A_{i-1}^{-1} u^{T} .
\end{aligned}
$$

Note that

$$
\left(\begin{array}{cc}
A_{i-1} & u^{T} \\
u & k_{i i}
\end{array}\right)
$$

is a principal submatrix of the positive-definite matrix $A$ and hence is positive definite. It follows that its final pivot $k_{i i}-u A_{i-1}^{-1} u^{T}$ is positive. Furthermore, since the negative Schur complement $S_{i-1}$ is positive definite, the second term in (2.3) is also positive. Therefore, $d_{i i}>0$.

Case 2: node $i$ is a $C$-node. In this case, we join the $i$-th row and column to the other $C$ nodes in the partition (2.2). This $i \times i$ matrix also satisfies the rank condition and thus its inverse is of the form (2.1). The $i$-th pivot is the reciprocal of the $(i, i)$ entry of the $i \times i$ principal submatrix, which in this case comes from the diagonal of the negative definite matrix $-S_{i-1}$. Thus $d_{i i}<0$.

As both cases give non zero pivots, by induction the ordering constraint ensures the $L D L^{T}$ factorization exists. Moreover, the pivots associated with the $A$-nodes are guaranteed to be positive and those associated with $C$-nodes are guaranteed to be negative. By rescaling, $L \leftarrow L|D|^{1 / 2}$ and $D \leftarrow \operatorname{sign}(D)=\operatorname{diag}( \pm 1)$, the diagonal matrix is fully determined in advance by the structure of the problem, independent of the numerical values. This gives a signed Cholesky factorization of $K$.

The signed Cholesky factorization allows Bridson to modify a Cholesky factorization code to perform the factorization of the indefinite matrix $K$ without numerical pivoting. A stability analysis is missing but Bridson reports that numerical experiments indicate, for well-scaled problems, the constrained ordering is generally sufficient to avoid numerical pivoting; this was supported by additional experiments given by Scott [54]. The hope is that, if an initial ordering is chosen to reduce fill in $L$, the additional fill that results from modifying the ordering to a constrained ordering will be modest. If the constrained ordering is close to the initial ordering, the potential benefits include a fast factorization and, importantly, the 
analyse phase of the direct solver can accurately predict the size of the factors and other data structures required during the numerical factorization.

For a direct solver, choosing a fill-reducing ordering is essential (both for the memory needed and for the flop count). Bridson proposed two approaches to computing a constrained ordering. The first modifies the minimum degree algorithm (or one of its variants) to incorporate the constraint within it. An alternative approach is to post-process a given fill-reducing ordering to satisfy the constraint. If a $C$-node is the next node in the supplied ordering, it is only included in the modified ordering once all its $A$-node neighbours have been ordered (that is, a $C$-node is postponed until after all its $A$-node neighbours). For many large problems, orderings based on nested dissection are frequently recommended in preference to those based on minimum degree. The advantages of the post-processing approach are that it can be applied to any fill-reducing ordering and it is very cheap and straightforward to implement. Hogg and Scott $[33,54]$ considered this approach and compared using it to compute a signed Cholesky factorization (with no pivoting for stability) with using a fill-reducing ordering with a solver designed to factorize indefinite matrices with threshold pivoting incorporated for stability. Their reported results showed that the constrained ordering leads to significantly denser factors and higher flop counts so that it is unlikely to be competitive in practice for a direct solver.

The situation in the incomplete case is potentially somewhat different since, while it is known that the ordering used can be important (see, for example, the numerical results reported in [56] and the references therein), the choice of ordering is generally less crucial than in the complete factorization. This is because, in the incomplete case, the number of entries in the factor is determined not by the ordering but by userdefined parameters that may include the amount of fill allowed in each column and drop tolerances. Thus our aim is to combine using a constrained ordering with a (modified) $I C$ factorization code to compute a signed $I C$ factorization that we can use with an iterative solver.

We remark that Rehman et al [61] propose an a priori ordering of the nodes in their development of incomplete factorization preconditioners for the (unsymmetric) saddle-point systems that arise from the finite-element discretization of incompressible Navier-Stokes equations. They employ a level-based bandor profile-reduction ordering, which they post-process level-by-level so that, at each level, the $A$-nodes precede the $C$-nodes.

\section{A limited-memory $I C$ factorization}

We now summarize the limited-memory $I C$ factorization algorithm that is implemented within the HSL package HSL_MI28. We assume here that $A$ is a symmetric and positive-definite matrix for which an $I C$ factorization is required. For such $A$, HSL_MI28 computes an $I C$ factorization $(Q L)(Q L)^{T}$, where $Q$ is a permutation matrix, chosen to preserve sparsity. The matrix $A$ is optionally scaled and, if necessary, following the approach of Manteuffel [41], shifted to avoid breakdown of the factorization. Thus the incomplete factorization of $\bar{A}=S Q^{T} A Q S+\alpha I$ is computed, where $S=\left\{s_{i}\right\}$ is a diagonal scaling matrix and $\alpha$ is a positive shift. The user supplies the lower triangular part of $A$ in compressed sparse column format and the computed $L$ is returned to the user in the same format; a separate entry performs the preconditioning operation $y=P z$, where $P=\left(\bar{L} \bar{L}^{T}\right)^{-1}, \bar{L}=Q S^{-1} L$, is the incomplete factorization preconditioner.

The algorithm implemented by HSL_MI28 is a limited memory Tismentsky-Kaporin approach. The Tismenetsky scheme is based on a matrix factorization of the form

$$
\bar{A}=(L+R)(L+R)^{T}-E,
$$

where $L$ is a lower triangular matrix with positive diagonal entries that is used for preconditioning, $R$ is a strictly lower triangular matrix with small entries that is used to stabilize the factorization process, and $E$ has the structure

$$
E=R R^{T}
$$


The Tismenetsky incomplete factorization does not compute the full update and thus a positive semidefinite modification is implicitly added to $A$.

Following the ideas of Kaporin [35], HSL MA28 uses drop tolerances to limit the memory required in the computation of the incomplete factorization. The user controls the dropping of small entries from $L$ and $R$ and the maximum number of entries within each column of $L$ and $R$ (and thus the amount of memory for $L$ and the intermediate work and memory used in computing the incomplete factorization). The parameters lsize and rsize must be set by the user to the maximum number of fill entries in each column of $L$ and the number of entries in $R$, respectively. Further details are given in [56, 57].

We present a summary outline of our left-looking incomplete factorization as Algorithm 3.1. It shows the basic steps but, for simplicity, omits details of our sparse implementation. Here $A_{:, j}, L_{:, j}$ and $R_{:, j}$ denote the $j$-th columns of the lower triangular parts of $A, L$ and $R$, respectively, and $n_{j}$ is the number of entries in column $j$ of $A$. The scalar small is used to determine whether a diagonal entry is sufficiently large; if at any stage a diagonal entry is less than small, the factorization is considered to have broken down and in this case, the shift $\alpha$ is increased and the factorization restarted. droptol $1>$ droptol $2 \geq 0$ are chosen drop tolerances. The user may choose to supply a positive initial shift $\alpha_{i n}$.

The factorization proceeds column-by-column. At each stage $j \geq 2$, updates from the previously computed columns 1 to $j-1$ of $L$ and $R$ are applied to column $j$ of $A$. The update operations are as follows:

for $k<j$ and $L_{j, k} \neq 0$ do

$$
\begin{aligned}
A_{j: n, j} & \Leftarrow A_{j: n, j}-L_{j: n, k} * L_{j, k} & & \text { ! L L } L^{T} \text { updates } \\
A_{j: n, j} & \Leftarrow A_{j: n, j}-R_{j: n, k} * L_{j, k} & & \text { ! RL updates }
\end{aligned}
$$

end

for $k<j$ and $R_{j, k} \neq 0$ do

end

$$
A_{j: n, j} \Leftarrow A_{j: n, j}-L_{j: n, k} * R_{j, k} \quad \text { ! L } L R^{T} \text { updates }
$$

The diagonal entries of columns $j+1$ to $n$ are also updated. This allows us to detect a potential breakdown as soon as possible. Once a breakdown is detected, the shift $\alpha$ is increased (the strategy for doing this is explained in [56]) and then the factorization of the shifted matrix $A+\left(\alpha-\alpha_{0}\right) I$ is restarted, where $\alpha_{0}$ is the previous shift.

Algorithm 3.1. Outline of the HSL_MI28 incomplete Cholesky algorithm for positive-definite $A$

Input: Symmetric positive definite $A \in R^{n \times n}$;

lsize, rsize, droptol1, droptol2, initial shift $\alpha_{\text {in }}$

Output: Incomplete Cholesky factor L, final shift $\alpha_{\text {out }}$

Compute a sparsity-preserving ordering $Q$ for $A$ and permute: $A \leftarrow Q^{T} A Q$

Compute a diagonal scaling $S$ and scale: $A \leftarrow S A S$

Set breakdown $=$ false and $\alpha=\alpha_{i n}, \alpha_{0}=0$

Loop over shifts

do

Set $A \leftarrow A+\left(\alpha-\alpha_{0}\right) I \quad$ and $\quad d(1: n)=\left(a_{11}, a_{22}, \ldots, a_{n n}\right)$

for $j=1: n$ do

Apply $L L^{T}, R L^{T}$ and $L R^{T}$ updates from columns $1: j-1$ to $A_{:, j}$ and $d(j+1: n)$

if $\min (d(j+1: n)<$ small then

Set breakdown $=$ true, $\alpha_{0}=\alpha$ and increase $\alpha$

exit (Restart factorization with larger shift) 
end if

Sort entries of $A_{:, j}$ by magnitude

Keep $n_{j}+$ lsize entries of largest magnitude in $L_{:, j}$, provided each is at least droptol 1

Keep the rsize entries that are next largest in magnitude in $R_{:, j}$, provided each is at least droptol 2

end do

if breakdown $=$ false then

Set $\alpha_{\text {out }}=\alpha-\alpha_{0}$ and return (Breakdown-free factorization complete) end if

end do

Note that the widely-used ICFS code of Lin and Moré [40] implements a special case in which ordering is not incorporated, droptol $1=$ droptol $2=0$ and rsize $=0$ (so that there is no dropping of entries by size, no intermediate memory is used and only $L L^{T}$ updates are applied).

\section{Signed incomplete Cholesky factorization}

We now discuss how to modify Algorithm 3.1 for saddle-point systems while avoiding the need to incorporate pivoting. Our signed $I C$ factorization is listed as Algorithm 4.1 (again, the sparse implementation details are omitted). Here $N_{j}$ is the number of entries in column $j$ of $K$. This algorithm is implemented within a new package HSL_MI30.

Algorithm 4.1. Outline of the HSL_MI30 signed incomplete Cholesky algorithm for saddle-point $K$ Input: Symmetric saddle-point matrix $K \in R^{N \times N}$ with $N=n+m$;

lsize, rsize, droptol1, droptol2, initial shifts $\alpha_{i n}(1: 2)$

Output: Incomplete signed Cholesky factor $L$, diagonal $D$ with $n$ positive and $m$ negative entries, final shifts $\alpha_{\text {out }}(1: 2)$

Compute a sparsity-preserving ordering $Q$ for $K$

Post-process the ordering $\hat{Q} \leftarrow Q \quad$ (see Section 2)

Permute the matrix: $K \leftarrow \hat{Q}^{T} K \hat{Q}$

Compute a diagonal scaling $S$ and scale: $K \leftarrow S K S$

Set breakdown $=$ false and $\alpha(1: 2)=\alpha_{\text {in }}(1: 2), \alpha_{0}(1: 2)=0$

Loop over shifts

do

Set $K \leftarrow K+G$ where $G$ is diagonal with $G_{i i}= \begin{cases}\alpha(1)-\alpha_{0}(1) & \text { if } i \text { is an } A \text { node } \\ -\alpha(2)+\alpha_{0}(2) & \text { if } i \text { is a } C \text { node }\end{cases}$

Set $d(1: N)=\left(k_{11}, k_{22}, \ldots, k_{N N}\right)$

for $j=1: N$ do

Apply $L L^{T}, R L^{T}$ and $L R^{T}$ updates from columns $1: j-1$ to $K_{:, j}$ and $d(j+1: N)$

if $\min (d(i): i$ is an $A$ node $)<$ small then

Set breakdown $=$ true, $\alpha_{0}(1: 2)=\alpha(1: 2)$ and increase $\alpha(1)$

exit (Restart factorization with larger $\alpha(1))$

else if $\max (d(i): i$ is a $C$ node $)>-$ small then 


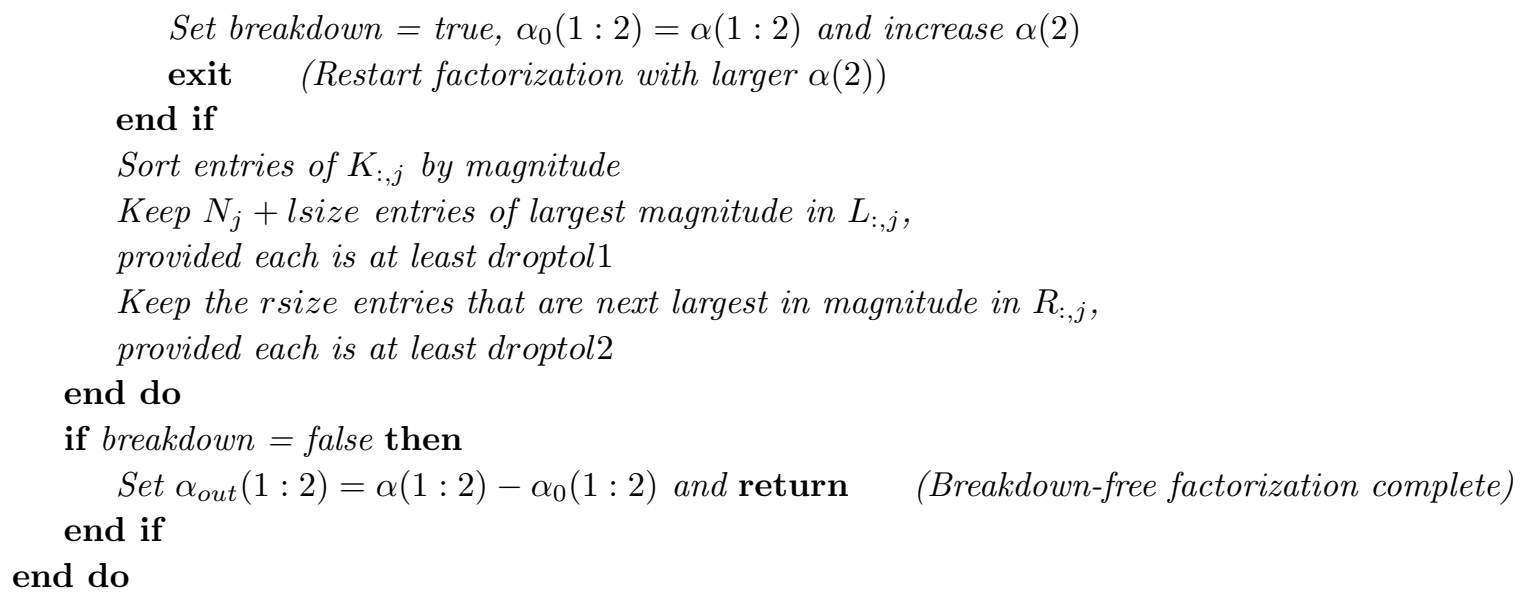

In addition to the step that post-processes the computed ordering so that a $C$-node is never ordered before its $A$-node neighbours, the key difference between the two algorithms is that, for the saddle-point case, we employ two shifts, $\alpha(1: 2)$. Both are non-negative. The first, $\alpha(1)$, is increased each time the incomplete factorization breaks down on an $A$-node while the second, $\alpha(2)$ is increased each time the incomplete factorization breaks down on a $C$-node. For simplicity of notation, assuming the natural ordering is used (so that $Q=I$ ), the incomplete factorization of the scaled and shifted matrix

$$
\bar{K}=S\left(\begin{array}{cc}
A & B^{T} \\
B & -C
\end{array}\right) S+\left(\begin{array}{cc}
\alpha(1) I & 0 \\
& -\alpha(2) I
\end{array}\right)
$$

is computed. We note that, recently, Chen et al [11] used a perturbation of the $(2,2)$ block in a zero-level fill incomplete factorization; they refer to this as a stabilized incomplete factorization. In their study, they use a fixed shift $\alpha(2)=-4$ (with $\alpha(1)=0$ ). Furthermore, in their paper on incomplete factorization preconditioners for Navier-Stokes solvers, Rehman et al [61] comment that convergence can sometimes be improved by perturbing the $(2,2)$ block.

It is clear that the changes that must be made to the Cholesky code are minimal; it is essentially only necessary to distinguish between $A$ and $C$-nodes. We observe that we did experiment with using a single shift (so that $\alpha(1)=\alpha(2)$ ) but found that this gave us significantly poorer results.

Note that this algorithm differs from that described in Section 1 since, even with the natural ordering, we do not compute an incomplete factorization of $A$ on its own and then solve (1.3) before computing an incomplete factorization of the approximate Schur complement (1.4). Rather, we work with the columns of length $N=n+m$ of the matrix

$$
\left(\begin{array}{c}
A \\
B
\end{array}\right)
$$

This has the advantage that the sparsity of the $(2,1)$ block of the incomplete factor is dealt with as the factorization of the $(1,1)$ block proceeds. Furthermore, it is not necessary to limit the factorization exclusively to the $A$-nodes before proceeding to the $C$-nodes; we only require that a $C$-node is ordered after all its $A$-node neighbours. This gives greater flexibility in the ordering of the factorization.

Finally, we remark that Orban [45] has recently proposed a limited-memory incomplete $L D L^{T}$ factorization for the important class of SQD matrices. His algorithm is a generalization of that of Lin and Moré [40]. It can be regarded as a special case of Algorithm 4.1 with droptol $1=$ droptol $2=0$, rsize $=0$ and $\alpha(1)=\alpha(2)$ and, as it is for SQD matrices, there is no requirement to post-process the sparsity-preserving ordering. 


\section{$5 \quad$ Theoretical results}

This section presents some results that may lead to a better theoretical understanding of limited memory incomplete factorizations. Our results closely follow those in $[44,45]$. However, unlike the SQD case that was recently considered by Orban [45], for our saddle-point systems, we cannot assume that the matrices have non zero diagonal entries and so we are not able to exploit the results summarized, for example, in [7].

Early analysis of incomplete factorizations was built on theory for special matrices, such as $M$ - and $H$-matrices, that correspond naturally to the early stages of solving linear systems by preconditioned iterative methods. For example, the proof of the breakdown-free property of incomplete factorizations for $M$-matrices is an important component of the seminal paper of Meijerink and van der Vorst [43] (see also $[41,66])$.

Definition 5.1. A non singular real square matrix $A$ with non positive off diagonal entries is called an $M$-matrix if all the entries of its inverse are non negative. A non singular real square matrix $A$ is called an $H$-matrix if its comparison matrix $M(A)=\left\{M(A)_{i j}\right\}$ defined as

$$
M(A)_{i j}= \begin{cases}\left|a_{i j}\right| & (i=j) \\ -\left|a_{i j}\right| & (i \neq j)\end{cases}
$$

is an $M$-matrix.

Note that $H$-matrices are very close to strictly diagonally dominant matrices [7] (they are so-called generalized diagonally dominant matrices). The following basic result can be found in [7].

Lemma 5.1. The Schur complement of an $M$-matrix (respectively, an $H$-matrix) is an $M$-matrix (respectively, an $H$-matrix). Furthermore, for real square matrices, if $A$ is an $H$-matrix and elementwise $B$ satisfies $M(B) \geq M(A)$, then $B$ is also an $H$-matrix.

We start by considering a single step of three possible factorization approaches: the complete factorization (which we denote as CO), the limited memory approach of Lin and Moré and Orban (LM) $[40,45]$, and our proposed incomplete symmetric Tismenetsky approach (TI). Assuming $d_{1}=a_{11}$ is non zero, we can write one step of the factorization of a symmetric matrix $A$ in the form

$$
A=\left(\begin{array}{cc}
d_{1} & w^{T} \\
w & F
\end{array}\right) \equiv\left(\begin{array}{ccc}
d_{1} & l^{T} & r^{T} \\
l & F_{L L} & F_{L R} \\
r & F_{R L} & F_{R R}
\end{array}\right) .
$$

For a CO factorization, we have the Schur complement

$$
S_{C O}=F-d_{1}^{-1}\left(\begin{array}{l}
l \\
r
\end{array}\right)\left(\begin{array}{ll}
l^{T} & r^{T}
\end{array}\right) .
$$

The LM factorization keeps only part of the pivot column (part $l$ in (5.1), assuming the rows that correspond to the retained entries are ordered first). Further, Lin and Moré update only $F_{L L}$ and, importantly, the diagonal entries of $F_{R R}$. The TI approach updates $F_{L L}, F_{R L}$ and $F_{L R}$ and possibly also the diagonal entries of $F_{R R}$. The following result is closely related to results in [40, 44, 45].

Lemma 5.2. Let $A$ be a symmetric $H$-matrix with $d_{1}=a_{11}$ non zero. Denote the Schur complements for the CO, LM and TI factorizations after one step with pivot d by $S_{C O}, S_{L M}$ and $S_{T I}$, respectively. Further, let the Schur complement of $M(A)$ be $S_{M(A)}$. Then, element-wise, $S_{M(A)}$ is not larger than any of $M\left(S_{C O}\right), M\left(S_{T I}\right)$ and $M\left(S_{L M}\right)$.

Proof: From (5.1), the comparison matrix of $A$ is

$$
M(A)=\left(\begin{array}{ccc}
\left|d_{1}\right| & -|l|^{T} & -|l|^{T} \\
-|l| & M\left(F_{L L}\right) & M\left(F_{L R}\right) \\
-|r| & M\left(F_{R L}\right) & M\left(F_{R R}\right)
\end{array}\right) .
$$


After one step of the $\mathrm{CO}$ factorization, the Schur complement of $M(A)$ is equal to

$$
S_{M(A)}=\left(\begin{array}{ll}
M\left(F_{L L}\right)-\left|d_{1}^{-1}\right||l||l|^{T} & M\left(F_{L R}\right)-\left|d_{1}^{-1} \| l\right||r|^{T} \\
M\left(F_{R L}\right)-\left|d_{1}^{-1}\|r\| l\right|^{T} & M\left(F_{R R}\right)-\left|d_{1}^{-1}\|r\| r\right|^{T}
\end{array}\right)
$$

and from (5.2), the comparison matrix of the Schur complement of $A$ is

$$
M\left(S_{C O}\right)=M\left(F-d_{1}^{-1}\left(\begin{array}{l}
l \\
r
\end{array}\right)\left(\begin{array}{ll}
l^{T} & r^{T}
\end{array}\right)\right)
$$

Setting $s=\left\{s_{i}\right\}=\left(\begin{array}{l}l \\ r\end{array}\right)\left(\begin{array}{ll}l^{T} & r^{T}\end{array}\right)$, the entries of $M\left(S_{C O}\right)$ can be expressed as

$$
M\left(S_{C O}\right)_{i j}= \begin{cases}\left|f_{i i}-d_{1}^{-1} s_{i}^{2}\right| & i=j \\ -\left|f_{i j}-d_{1}^{-1} s_{i} s_{j}\right| & i \neq j\end{cases}
$$

Using the triangle inequality element-wise for the diagonal and the off diagonal entries separately, we obtain

$$
\left|f_{i i}-d_{1}^{-1} s_{i}^{2}\right| \geq\left|f_{i i}\right|-\left|d_{1}\right|^{-1} s_{i}^{2}
$$

and

$$
-\left|f_{i j}-d_{1}^{-1} s_{i} s_{j}\right| \geq-\left|f_{i j}\right|-\left|d_{1}\right|^{-1}\left|s_{i}\right|\left|s_{j}\right|
$$

for $i=j$ and $i \neq j$, respectively. From (5.3) it follows that $S_{M(A)} \leq M\left(S_{C O}\right)$ element-wise. Consider now the comparison matrices $M\left(S_{T I}\right)$ and $M\left(S_{L M}\right)$. The Schur complement update in both replaces some of the off diagonal updates $s_{i} s_{j}$ by zeros because of dropping. But even in this case the inequalities (5.4) and (5.5) remain valid and the required result may be deduced. Note that the proof does not provide a partial ordering among off diagonal entries of $M\left(S_{C O}\right), M\left(S_{T I}\right)$ and $M\left(S_{L M}\right)$.

Results of this kind for $M$ - or $H$-matrices, as well as stronger results that typically assume non zero diagonal entries, are useful as well as popular. However, the fact that we can get such results for a wide class of incomplete factorization methods potentially indicates that the resulting accuracy may be poor [12]. In the other words, lower bounds for the sequence of the Schur complements may be weak and we may obtain a factorization for a problem that is far from the original. We also know that the size of the modification may determine the efficiency of the preconditioned iterative method $[18,21,56]$, but see the note in [40] stating that it is not clear whether this conclusion transfers to limited memory preconditioners. We conclude that it is still necessary to have a good choice of incomplete factorization and its implementation. This idea will hopefully remain a driving force for the development new approaches.

We now consider the TI factorization of the saddle-point matrix (1.1) (without drop tolerances).

Theorem 5.1. Consider the incomplete TI factorization of the symmetric saddle-point matrix (1.1), with a pivot sequence such that a $C$-node is only ordered after all its A-node neighbours. Assume that for an $A$-pivot, all its $C$-node neighbours are in $L$ and for a $C$-pivot, all its $C$-node neighbours are in $R$. Then all the entries of the diagonal factor $D$ are \pm 1 and the factorization is breakdown-free.

Proof: Assume $i-1$ steps have succeeded and consider the $i$-th pivot $k_{i i}$. As in Theorem 2.1, we consider the partitioned $i$-th principal submatrix

$$
\left(\begin{array}{ccc}
A_{i-1} & B_{i-1}^{T} & u^{T} \\
B_{i-1} & -C_{i-1} & v^{T} \\
u & v & k_{i i}
\end{array}\right) .
$$

Recall that, at each step, the TI factorization is based on adding a positive semidefinite matrix to the matrix that is being factorized (see [57]). Under the stated assumptions, at the $i$-th step a positive semidefinite matrix has only been added to $A_{i-1}$; let $\Delta$ denote this positive semidefinite matrix. 
Case 1: node $i$ is a $A$-node. In this case, the proof is very similar to that of the complete factorization. As in Theorem 2.1, the ordering implies that $v$ is equal to zero. Further, the update of $k_{i i}$ includes just two terms as in (2.3). The first of these terms equal to $u\left(A_{i-1}+\Delta\right)^{-1} u^{T}$. Again, as in (2.3), the second term updating $k_{i i}$ is based on the Schur complement $S_{i-1}=-C_{i-1}-B_{i-1}\left(A_{i-1}+\Delta\right)^{-1} B_{i-1}^{T}$ and it is equal to $-u\left(A_{i-1}+\Delta\right)^{-1} B_{i-1}^{T} S_{i-1}^{-1} B_{i-1}\left(A_{i-1}+\Delta\right)^{-1} u^{T}$ and thus it is also positive.

Case 2: node $i$ is a $C$-node. The update of $k_{i i}$ can be split into two steps. First, we subtract from $k_{i i}$ the positive value $u\left(A_{i-1}+\Delta\right)^{-1} u^{T}$ and so $k_{i i}$ remains negative. Second, $k_{i i}$ should be updated using the Schur complement $S_{i-1}=-C_{i-1}-B_{i-1}\left(A_{i-1}+\Delta\right)^{-1} B_{i-1}^{T}$. But $S_{i-1}$ corresponds to $C$-nodes and, because of the assumption that all $C$-node neighbours of a $C$-pivot are in $R$ and the TI factorization does not perform $R R^{T}$ updates, $k_{i i}$ is not updated further and is thus negative.

Remark 5.1. The result in Theorem 5.1 proposes a modification of the signed factorization that safeguards the existence of the IC factorization, even for saddle-point matrices with zero diagonal entries. Note that, in practice, when developing a fill-in efficient implementation, we generally need to relax the theoretical conditions. For example, the diagonal entries of the $(2,2)$ block could be modified by adding a negative semidefinite (diagonal) matrix.

Remark 5.2. Theorem 5.1 does not hold for the LM factorization, even when $K$ is a symmetric saddlepoint $H$-matrix. In this case, even when we have the monotonicity property in the sequence of the Schur complements as described in Lemma 5.2, there is no guarantee that the diagonal $C$-node entries will be non zero.

For the LM factorization, we require more stringent assumptions.

Theorem 5.2. Assume that the symmetric saddle-point matrix (1.1) is an H-matrix and that the constraint ordering is as in Theorem 5.1. Assume further that all the A-nodes are fully updated, that is, they correspond to the CO factorization. Then the LM factorization that updates all the diagonal entries at each stage is breakdown-free.

Proof: The complete factorization of the $A$-nodes with the diagonal updates of all the nodes is necessary to ensure the diagonal $C$-node entries become nonzero. The $H$-matrix property explained in Lemma 5.2 can then be applied for all the subsequent Schur complements.

While the theoretical results presented here appear promising, as already observed, practical implementations almost invariably employ further relaxations and extend solvability from (relatively weak, as we mentioned above) special matrices to more general ones. The real challenge is thus (1) to minimize the fill-in in the computed factors, (2) to use as much information on the problem as possible for the computation, and (3) to restrict the memory use. The breakdown-free property can then be forced by scaling and moving the scaled matrix closer to a related $H$-matrix through the use of diagonal shifts [40].

\section{$6 \quad$ Numerical experiments}

\subsection{Test environment}

All the numerical results reported on in this paper are performed (in serial) on our test machine that has two Intel Xeon E5620 processors with 24 GB of memory. The ifort Fortran compiler (version 12.0.0) with option -O3 is used. The implementations of the SYMMBK algorithm and the GMRES(100) algorithm (with right preconditioning) offered by the HSL routines HSL_MI02 and MI24, respectively, are employed, with starting vector $x_{0}=0$, the right-hand side vector $b$ computed so that the exact solution is $x=1$, and stopping criteria

$$
\|K \hat{x}-b\|_{2} \leq 10^{-8}\|b\|_{2},
$$


where $\hat{x}$ is the computed solution. In addition, for each test we impose a limit of 1000 iterations.

Our test problems are real indefinite matrices taken from the University of Florida Sparse Matrix Collection [14]. The first set (Test Set 1) has zero $(2,2)$ block $(C=0)$; they are listed in Table 6.1 . The second set (Test Set 2) are interior-point optimization matrices with $C=10^{-8} I$; they are listed in Table 6.2. Here we give the order $n$ of the $(1,1)$ block $A$, the order $m$ of the $(2,2)$ block $C$ and the number $n z(K)$ of entries in the lower triangular part of $K$. In addition, we use the direct solver HSL_MA97 [32] to compute the number of entries in the complete factor of $K$. For the HSL_MA97 runs, we use the scaling from a symmetrized version of the package MC64 [17, 19]. We remark that this scaling has been found to work well for direct solvers when used to solve "tough" indefinite systems [30, 33]. We also use the default ordering (which is either approximate minimum degree or nested dissection, and this is selected automatically following [20]), except for the problems marked *. For these, we found we obtain a sparser complete factorization by using a matching-based ordering. We report $f_{i l l_{L}}$ to be the ratio of the number of entries in the factor to $n z(K)$. This statistic is reported for later comparison with the fill for the incomplete factorizations.

Table 6.1: Test problems with $C=0$ (Test Set 1). $n$ and $m$ denote the order of $A$ and $C$ (see 1.1$), n z(K)$ is the number of entries in the lower triangular part of $K, f_{i l l}$ is the ratio of is the number of entries in the complete factor of $K$ to $n z(K)$. QP $=$ quadratic programming problem, $\mathrm{FE}=$ finite-element, $\mathrm{PDE}=$ partial differential equation. ${ }^{*}$ denotes matching-based ordering is used.

\begin{tabular}{lrrrll}
\hline \multicolumn{1}{c}{ Identifier } & \multicolumn{1}{c}{$n$} & \multicolumn{1}{c}{$n z(K)$} & $f i l_{L}$ & Description/Application \\
\hline GHS_indef/aug3dcqp & 27543 & 8000 & 77829 & 18.3 & 3D PDE \\
GHS_indef/boyd1 & 93261 & 18 & 652246 & 1.00 & Convex QP \\
GHS_indef/brainpc2* & 13807 & 13800 & 96601 & 3.61 & Biological model \\
GHS_indef/cont-201* & 40397 & 40198 & 239596 & 20.1 & Convex QP \\
GHS_indef/cont-300* & 90597 & 90298 & 539396 & 22.1 & Convex QP \\
GHS_indef/d_pretok & 129160 & 53570 & 885416 & 17.3 & Mixed FE model \\
\hline GHS_indef/darcy003 & 234128 & 155746 & 1167685 & 7.86 & Mixed FE model \\
GHS_indef/mario001 & 23130 & 15304 & 114643 & 6.51 & FE model Stokes problem \\
GHS_indef/ncvxqp9* & 9004 & 7500 & 31547 & 11.4 & Nonconvex QP \\
GHS_indef/oslenski0 & 61030 & 27233 & 402623 & 12.1 & Mixed FE model \\
GHS_indef/qpband & 15000 & 5000 & 30000 & 1.67 & Convex QP \\
GHS_indef/sit100 & 7142 & 3120 & 34094 & 13.7 & Mixed FE model \\
\hline GHS_indef/stokes64 & 8450 & 4096 & 74242 & 8.98 & FE model Stokes problem \\
GHS_indef/stokes128 & 33282 & 16384 & 295938 & 10.7 & FE model Stokes problem \\
GHS_indef/tuma1 & 13360 & 9607 & 50560 & 10.9 & Mixed FE model \\
GHS_indef/tuma2 & 7515 & 5477 & 28440 & 10.5 & Mixed FE model \\
GHS_indef/turon_m & 133814 & 56110 & 912345 & 15.7 & Mixed FE model \\
\hline & & & & & \\
\end{tabular}

Following [55], in our experiments we define the efficiency of the preconditioner $P$ to be

$$
\text { efficiency }=\text { iter } \times n z(L)
$$

where iter is the iteration count for $P=\left(L D L^{T}\right)^{-1}$. The lower the value of $(6.2)$, the better the preconditioner. We also define the fill in the incomplete factor to be the ratio

$$
f i l l_{I C}=(\text { number of entries in the incomplete factor }) / n z(K) \text {, }
$$

\subsection{Results for $C=0$}

In Table 6.3, we present results for Test Set 1. The values of the shifts $\alpha_{\text {out }}(1: 2)$ are reported, together with fill $_{I C}$, the efficiency and the number of iterations. We use the natural ordering (so that all $A$-nodes are ordered ahead of the $C$-nodes) and symmetrized MC64 scaling. The drop tolerances are $d r o p t o l 1=10^{-3}$ and droptol $2=10^{-4}$ (which are the default values for HSL_MI28). We ran GMRES(100) for each problem 
Table 6.2: Interior-point test problems (Test Set 2). $n$ and $m$ denote the order of $A$ and $C$ (see 1.1), $n z(K)$ is the number of entries in the lower triangular part of $K, f_{i l l} L$ is the ratio of is the number of entries in the complete factor of $K$ to $n z(K)$.

\begin{tabular}{lrrrl}
\hline \multicolumn{1}{c}{ Identifier } & $n$ & $m$ & $n z(K)$ & $f_{i l l_{L}}$ \\
\hline GHS_indef/c-55 & 19121 & 13659 & 218115 & 21.5 \\
GHS_indef/c-59 & 23813 & 17469 & 260909 & 17.6 \\
GHS_indef/c-63 & 25505 & 18729 & 239469 & 13.7 \\
GHS_indef/c-68 & 36546 & 28264 & 315408 & 29.2 \\
GHS_indef/c-69 & 38432 & 29026 & 345714 & 10.9 \\
GHS_indef/c-70 & 39302 & 29622 & 363955 & 13.7 \\
GHS_indef/c-71 & 44814 & 31824 & 468096 & 37.1 \\
GHS_indef/c-72 & 47950 & 36114 & 395811 & 11.7 \\
Schenk_IBMNA/c-big & 201877 & 143364 & 1343126 & 39.0 \\
\hline
\end{tabular}

with lsize $=$ rsize $=1,5,10$ and 20 and selected the best result (in terms of the lowest value of the efficiency (6.2)). For problems GHS_indef/cont-201 and GHS_indef/cont-300, we did not achieve convergence with these settings but did get convergence by choosing droptol $1=$ droptol $2=0.0$, lsize $=$ rsize $=20$ and running SYMMBK.

Table 6.3: GMRES(100) convergence results for Test Set 1. * indicates SYMMBK was used.

\begin{tabular}{lrrrcrrr}
\hline \multicolumn{1}{c}{ Identifier } & lsize & rsize & $\alpha_{\text {out }}(1)$ & $\alpha_{\text {out }}(2)$ & $f_{l l} l_{I C}$ & efficiency & iters \\
\hline GHS_indef/aug3dcqp & 1 & 1 & 0.0 & 0.0 & 1.35 & $1.0 \times 10^{5}$ & 1 \\
GHS_indef/boyd1 & 1 & 1 & 0.0 & 0.0 & 0.82 & $3.8 \times 10^{6}$ & 7 \\
GHS_indef/brainpc2 & 1 & 1 & 0.0 & $2.56 \times 10^{-1}$ & 1.28 & $1.6 \times 10^{7}$ & 132 \\
GHS_indef/cont-201* & 20 & 20 & 0.0 & $1.00 \times 10^{-3}$ & 7.89 & $3.9 \times 10^{8}$ & 207 \\
GHS_indef/cont-300* & 20 & 20 & 0.0 & $1.00 \times 10^{-3}$ & 7.87 & $1.6 \times 10^{9}$ & 390 \\
GHS_indef/d_pretok & 5 & 5 & 0.0 & $2.56 \times 10^{-1}$ & 1.96 & $4.8 \times 10^{7}$ & 28 \\
\hline GHS_indef/darcy003 & 20 & 20 & 0.0 & 0.0 & 5.44 & $2.8 \times 10^{8}$ & 44 \\
GHS_indef/mario001 & 20 & 20 & 0.0 & 0.0 & 5.37 & $9.9 \times 10^{6}$ & 16 \\
GHS_indef/ncvxqp9 & 10 & 10 & 0.0 & $4.19 \times 10^{3}$ & 4.01 & $2.5 \times 10^{5}$ & 2 \\
GHS_indef/olesnik0 & 20 & 20 & 0.0 & 0.0 & 4.43 & $4.8 \times 10^{7}$ & 27 \\
GHS_indef/qpband & 1 & 1 & 0.0 & 0.0 & 1.17 & $3.5 \times 10^{4}$ & 1 \\
GHS_indef/sit100 & 20 & 20 & 0.0 & 0.0 & 3.61 & $1.7 \times 10^{6}$ & 14 \\
\hline GHS_indef/stokes64 & 10 & 10 & 0.0 & 0.0 & 2.73 & $3.2 \times 10^{7}$ & 157 \\
GHS_indef/stokes128 & 10 & 10 & 0.0 & 0.0 & 2.73 & $4.3 \times 10^{8}$ & 539 \\
GHS_indef/tuma1 & 20 & 20 & 0.0 & 0.0 & 5.79 & $5.9 \times 10^{6}$ & 20 \\
GHS_indef/tuma2 & 20 & 20 & 0.0 & 0.0 & 6.55 & $3.4 \times 10^{6}$ & 18 \\
GHS_indef/turon_m & 20 & 20 & 0.0 & $1.60 \times 10^{-2}$ & 4.36 & $2.2 \times 10^{8}$ & 56 \\
\hline
\end{tabular}

In the positive-definite case, we found that using intermediate memory (rsize $>0$ ) was beneficial $[56,57]$. To assess whether it is also helpful for the signed $I C$ factorization, we run with the same settings (except rsize $=0$ ) and report the results in Table 6.4. We observe that, with rsize $=0$, more problems require a non-zero shift $\alpha_{\text {out }}(2)$ and that the value of the non-zero $\alpha_{\text {out }}(2)$ is greater than for rsize $>0$. Furthermore, with the same lsize, the fill ratio $f_{i l l} I C$ is generally greater and the efficiency poorer. For a number of problems, including GHS_indef/brainpc2 and GHS_indef/stokes64, the iteration count is significantly higher with $r$ size $=0$ and for problems GHS_indef/cont-300 and GHS_indef/stokes128, we fail to achieve the requested accuracy within the limit of 1000 iterations. We conclude that, overall, using intermediate memory improves the quality of the incomplete factorization.

It may be important to limit the amount of fill so in Table 6.5 we present results with lsize $=5$, rsize $=10$. We omit the first six problems and GHS_indef/qpband because they either fail to converge with 
Table 6.4: GMRES(100) convergence results for Test Set 1 with $r$ size $=0$. * indicates SYMMBK was used. - denotes failure to converge within 1000 iterations.

\begin{tabular}{lrrrcrrr}
\hline \multicolumn{1}{c}{ Identifier } & lsize & rsize & $\alpha_{\text {out }}(1)$ & $\alpha_{\text {out }}(2)$ & $f i l_{I C}$ & efficiency & iters \\
\hline GHS_indef/aug3dcqp & 1 & 0 & 0.0 & 0.0 & 1.56 & $1.2 \times 10^{5}$ & 1 \\
GHS_indef/boyd1 & 1 & 0 & 0.0 & 0.0 & 0.82 & $3.8 \times 10^{6}$ & 7 \\
GHS_indef/brainpc2 & 1 & 0 & 0.0 & 4.10 & 1.43 & $7.2 \times 10^{7}$ & 519 \\
GHS_indef/cont-201* & 20 & 0 & 0.0 & $1.60 \times 10^{-2}$ & 7.89 & $1.3 \times 10^{9}$ & 710 \\
GHS_indef/cont-300* & 20 & 0 & 0.0 & $1.60 \times 10^{-2}$ & 7.87 & - & - \\
GHS_indef/d_pretok & 5 & 0 & 0.0 & 0.0 & 2.09 & $5.9 \times 10^{7}$ & 32 \\
\hline GHS_indef/darcy003 & 20 & 0 & 0.0 & 0.0 & 6.13 & $3.1 \times 10^{8}$ & 43 \\
GHS_indef/mario001 & 20 & 0 & 0.0 & 0.0 & 6.00 & $1.0 \times 10^{7}$ & 15 \\
GHS_indef/ncvxqp9 & 10 & 0 & 0.0 & $4.19 \times 10^{3}$ & 6.44 & $4.1 \times 10^{5}$ & 2 \\
GHS_indef/olesnik0 & 20 & 0 & 0.0 & 0.0 & 5.45 & $6.8 \times 10^{7}$ & 31 \\
GHS_indef/qpband & 1 & 0 & 0.0 & 0.0 & 1.17 & $3.5 \times 10^{4}$ & 1 \\
GHS_indef/sit100 & 20 & 0 & 0.0 & 0.0 & 4.36 & $2.4 \times 10^{6}$ & 16 \\
\hline GHS_indef/stokes64 & 10 & 0 & 0.0 & $1.60 \times 10^{-2}$ & 2.74 & $5.9 \times 10^{7}$ & 289 \\
GHS_indef/stokes128 & 10 & 0 & 0.0 & $1.60 \times 10^{-2}$ & 2.73 & - & - \\
GHS_indef/tuma1 & 20 & 0 & 0.0 & $1.60 \times 10^{-2}$ & 8.22 & $6.6 \times 10^{6}$ & 16 \\
GHS_indef/tuma2 & 20 & 0 & 0.0 & $1.60 \times 10^{-2}$ & 7.93 & $3.2 \times 10^{6}$ & 14 \\
GHS_indef/turon_m & 20 & 0 & 0.0 & $2.56 \times 10^{-1}$ & 5.22 & $3.5 \times 10^{8}$ & 73 \\
\hline
\end{tabular}

Table 6.5: GMRES(100) convergence results for Test Set 1 with lsize $=5$, rsize $=10$.

\begin{tabular}{lrcrrr}
\hline \multicolumn{1}{c}{ Identifier } & $\alpha_{\text {out }}(1)$ & $\alpha_{\text {out }}(2)$ & fill $_{I C}$ & efficiency & iters \\
\hline GHS_indef/darcy003 & 0.0 & 0.0 & 2.69 & $1.1 \times 10^{9}$ & 349 \\
GHS_indef/mario001 & 0.0 & 0.0 & 2.68 & $2.7 \times 10^{7}$ & 89 \\
GHS_indef/ncvxqp9 & 0.0 & $1.05 \times 10^{3}$ & 3.34 & $3.2 \times 10^{5}$ & 3 \\
GHS_indef/olesnik0 & 0.0 & 0.0 & 2.13 & $6.9 \times 10^{7}$ & 81 \\
GHS_indef/sit100 & 0.0 & 0.0 & 2.12 & $4.0 \times 10^{6}$ & 55 \\
\hline GHS_indef/stokes64 & 0.0 & 0.0 & 1.90 & $2.9 \times 10^{7}$ & 204 \\
GHS_indef/stokes128 & 0.0 & 0.0 & 1.89 & $4.1 \times 10^{8}$ & 730 \\
GHS_indef/tuma1 & 0.0 & 0.0 & 2.78 & $8.3 \times 10^{6}$ & 59 \\
GHS_indef/tuma2 & 0.0 & 0.0 & 2.91 & $4.4 \times 10^{6}$ & 50 \\
GHS_indef/turon_m & 0.0 & 0.0 & 2.08 & $2.3 \times 10^{8}$ & 119 \\
\hline
\end{tabular}


these settings (problems GHS_indef/cont-201 and GHS_indef/cont-300) or the results already presented in Table 6.3 use less memory. We see that the fill is now less than 3.0 but, for some problems (including GHS_indef/darcy003 and GHS_indef/mario001), the quality of the preconditioner, in terms of the efficiency as well as the iteration count, is significantly poorer.

We have also considered varying lsize and rsize while keeping lsize + rsize constant. Results for a subset of our test problems for a range of pairs of values (lsize, rsize) are given in Table 6.6. We see that, as lsize increases, the level of fill increases and the iteration count reduces. However, as rsize increases, breakdown is avoided and the efficiency can improve. Thus we conclude that reducing the memory used for the factor while increasing the intermediate memory by a corresponding amount can be beneficial.

Table 6.6: GMRES(100) convergence results for problems from Test Set 1 with lsize + rsize $=20$.

\begin{tabular}{clrrrrr}
\hline Identifier & $($ lsize,rsize $)$ & $\alpha_{\text {out }}(1)$ & $\alpha_{\text {out }}(2)$ & fill $_{I C}$ & efficiency & iters \\
\hline GHS_indef/mario001 & $(20,0)$ & 0.0 & 0.0 & 6.00 & $1.0 \times 10^{7}$ & 15 \\
& $(15,5)$ & 0.0 & 0.0 & 4.71 & $1.3 \times 10^{7}$ & 24 \\
& $(10,10)$ & 0.0 & 0.0 & 3.84 & $1.5 \times 10^{7}$ & 35 \\
& $(5,15)$ & 0.0 & 0.0 & 2.68 & $2.7 \times 10^{7}$ & 87 \\
\hline GHS_indef/stokes64 & $(20,0)$ & 0.0 & 0.0 & 4.42 & $3.6 \times 10^{7}$ & 110 \\
& $(15,5)$ & 0.0 & 0.0 & 3.57 & $4.1 \times 10^{7}$ & 154 \\
& $(10,10)$ & 0.0 & 0.0 & 2.73 & $3.2 \times 10^{7}$ & 157 \\
& $(5,15)$ & 0.0 & 0.0 & 1.90 & $2.6 \times 10^{7}$ & 182 \\
\hline GHS_indef/tuma1 & $(20,0)$ & 0.0 & $1.60 \times 10^{-2}$ & 8.22 & $6.7 \times 10^{6}$ & 16 \\
& $(15,5)$ & 0.0 & 0.0 & 4.90 & $6.7 \times 10^{6}$ & 27 \\
& $(10,10)$ & 0.0 & 0.0 & 3.90 & $7.2 \times 10^{6}$ & 37 \\
& $(5,15)$ & 0.0 & 0.0 & 2.77 & $8.3 \times 10^{6}$ & 59 \\
\hline GHS_indef/turon_m & $(20,0)$ & 0.0 & $2.56 \times 10^{-1}$ & 5.22 & $3.5 \times 10^{8}$ & 73 \\
& $(15,5)$ & 0.0 & $2.56 \times 10^{-1}$ & 3.78 & $2.3 \times 10^{8}$ & 67 \\
& $(10,10)$ & 0.0 & 0.0 & 3.01 & $2.3 \times 10^{8}$ & 83 \\
& $(5,15)$ & 0.0 & 0.0 & 2.09 & $2.1 \times 10^{8}$ & 113 \\
\hline
\end{tabular}

In Table 6.7 we present results for different orderings. In each case, the ordering algorithm is applied to the sparsity pattern of $K$ and then post-processed, as described in Section 2. The lsize and rsize values are the same as in Table 6.3. The orderings tested are the profile reduction ordering of Sloan $[47,58,59]$ (as implemented by the HSL package MC61), Reverse Cuthill McKee (RCM) [13] (again, implemented within MC61), and approximate minimum degree (AMD) [3] (HSL_MC68). Generally, the Sloan ordering appears to be the best, although it did not lead to the required convergence for GHS_indef/brainpc2 and the tough GHS_indef/cont problems (for these, the natural order, which forces all the $C$-nodes after all the $A$-nodes, gives the best results).

In Table 6.8 we present results for different scalings. The scalings tested are symmetrized MC64, equilibration scaling using MC77 [50,51], and $l_{2}$ scaling, in which the entries in column $j$ of $K$ are normalised by the 2-norm of column $j$. We also report results for no scaling. We see that some problems are well-scaled and each of the three scalings has little effect on the iteration count (for example, GHS_indef/darcey003 and GHS_indef/mario001). However, whereas we achieved convergence in all cases using MC64 or MC77 scaling, we had a number of failures if no scaling was used and also we had failures with the $l_{2}$ scaling. While no strategy is consistently the best, in these tests, MC64 generally gives lower iteration counts than MC77 and the difference is sometimes large (for example, GHS_indef/brainpc2 and GHS_indef/stokes128).

\subsection{Results for $C=10^{-8} I$}

In Table 6.9, we present results for Test Set 2 (interior-point matrices). We use lsize $=$ rsize $=10$ with the Sloan ordering, symmetrized MC77 scaling and drop tolerances droptol $1=10^{-3}$ and droptol $2=10^{-4}$. In these tests, we use initial shifts $\alpha_{i n}(1: 2)=0.01$ to regularize the problem [52]. The iteration counts 
Table 6.7: GMRES(100) iteration results for Test Set 1 run with different orderings. The lowest iteration count for each problem is in bold. * indicates SYMMBK was used. - denotes failure to converge within 1000 iterations.

\begin{tabular}{lrrrrrr}
\hline \multicolumn{1}{c}{ Identifier } & lsize & rsize & Natural & Sloan & AMD & RCM \\
\hline GHS_indef/aug3dcqp & 1 & 1 & $\mathbf{1}$ & $\mathbf{1}$ & $\mathbf{1}$ & $\mathbf{1}$ \\
GHS_indef/boyd1 & 1 & 1 & 7 & $\mathbf{1}$ & 10 & 10 \\
GHS_indef/brainpc2 & 1 & 1 & $\mathbf{1 3 2}$ & - & - & 251 \\
GHS_indef/cont-201* & 20 & 20 & $\mathbf{2 0 7}$ & - & 741 & - \\
GHS_indef/cont-300* & 20 & 20 & $\mathbf{3 9 0}$ & - & - & - \\
GHS_indef/d_pretok & 5 & 5 & $\mathbf{2 8}$ & 93 & 122 & 96 \\
\hline GHS_indef/darcy003 & 20 & 20 & 44 & $\mathbf{3 7}$ & 46 & 46 \\
GHS_indef/mario001 & 20 & 20 & 16 & $\mathbf{1 4}$ & 17 & 16 \\
GHS_indef/ncvxqp9 & 10 & 10 & 2 & $\mathbf{1}$ & 2 & 2 \\
GHS_indef/olesnik0 & 20 & 20 & 27 & $\mathbf{2 3}$ & 27 & 27 \\
GHS_indef/qpband & 1 & 1 & $\mathbf{1}$ & $\mathbf{1}$ & $\mathbf{1}$ & $\mathbf{1}$ \\
GHS_indef/sit100 & 20 & 20 & 14 & $\mathbf{1 1}$ & 12 & 12 \\
\hline GHS_indef/stokes64 & 10 & 10 & 157 & $\mathbf{7 0}$ & 97 & 97 \\
GHS_indef/stokes128 & 10 & 10 & 539 & $\mathbf{1 9 7}$ & 444 & 444 \\
GHS_indef/tuma1 & 20 & 20 & 20 & $\mathbf{1 2}$ & 13 & $\mathbf{1 2}$ \\
GHS_indef/tuma2 & 20 & 20 & 18 & 12 & $\mathbf{1 1}$ & $\mathbf{1 1}$ \\
GHS_indef/turon_m & 20 & 20 & 56 & $\mathbf{3 5}$ & 48 & 38 \\
\hline
\end{tabular}

Table 6.8: GMRES(100) iteration results for Test Set 1 run with different scalings. The lowest iteration count for each problem is in bold. * indicates SYMMBK was used. - denotes failure to converge within 1000 iterations.

\begin{tabular}{lrrrcrr}
\hline \multicolumn{1}{c}{ Identifier } & lsize & rsize & MC64 & MC77 & $l_{2}$ & None \\
\hline GHS_indef/aug3dcqp & 1 & 1 & $\mathbf{1}$ & $\mathbf{1}$ & - & 79 \\
GHS_indef/boyd1 & 1 & 1 & 7 & 57 & 3 & $\mathbf{1}$ \\
GHS_indef/brainpc2 & 1 & 1 & 132 & 238 & 186 & $\mathbf{1 0 0}$ \\
GHS_indef/cont-201* & 20 & 20 & $\mathbf{2 0 7}$ & 246 & 209 & - \\
GHS_indef/cont-300* & 20 & 20 & $\mathbf{3 9 0}$ & 475 & - & - \\
GHS_indef/d_pretok & 5 & 5 & $\mathbf{2 8}$ & 41 & 44 & 144 \\
\hline GHS_indef/darcy003 & 20 & 20 & 44 & 44 & 42 & $\mathbf{4 1}$ \\
GHS_indef/mario001 & 20 & 20 & $\mathbf{1 6}$ & $\mathbf{1 6}$ & $\mathbf{1 6}$ & $\mathbf{1 6}$ \\
GHS_indef/ncvxqp9 & 10 & 10 & 2 & 1 & 1 & 1 \\
GHS_indef/olesnik0 & 20 & 20 & 27 & 28 & 27 & $\mathbf{2 3}$ \\
GHS_indef/qpband & 1 & 1 & $\mathbf{1}$ & $\mathbf{1}$ & $\mathbf{1}$ & $\mathbf{1}$ \\
GHS_indef/sit100 & 20 & 20 & 14 & 17 & 15 & $\mathbf{1 2}$ \\
\hline GHS_indef/stokes64 & 10 & 10 & 157 & 171 & $\mathbf{7 8}$ & 149 \\
GHS_indef/stokes128 & 10 & 10 & $\mathbf{5 3 9}$ & 980 & - & 568 \\
GHS_indef/tuma1 & 20 & 20 & 20 & 19 & $\mathbf{1 8}$ & $\mathbf{1 8}$ \\
GHS_indef/tuma2 & 20 & 20 & 18 & 17 & 17 & $\mathbf{1 6}$ \\
GHS_indef/turon_m & 20 & 20 & 56 & 52 & $\mathbf{5 0}$ & $\mathbf{5 0}$ \\
\hline
\end{tabular}


for the same settings but lsize $=10, r$ size $=0$ (no intermediate memory) are also reported. We see that using intermediate memory generally leads to a significant improvement in the preconditioner quality. In each case, our choice $\alpha_{i n}(2)=0.01$ was not large enough and we had to increase the $C$-shift and restart.

Table 6.9: GMRES(100) convergence results for Test Set 2 with lsize $=$ rsize $=10$ and $\alpha_{i n}(1: 2)=0.01$. The figures in parentheses are the iteration counts for lsize $=10$, rsize $=0$.

\begin{tabular}{llrrrrr}
\hline \multicolumn{1}{c}{ Identifier } & $\alpha_{\text {out }}(1)$ & $\alpha_{\text {out }}(2)$ & fill $_{I C}$ & efficiency & \multicolumn{2}{c}{ iters } \\
\hline GHS_indef/c-55 & 0.01 & 0.64 & 2.08 & $5.3 \times 10^{7}$ & 117 & $(147)$ \\
GHS_indef/c-59 & 0.01 & 0.64 & 2.10 & $6.0 \times 10^{7}$ & 110 & $(129)$ \\
GHS_indef/c-63 & 0.01 & 0.64 & 2.30 & $4.8 \times 10^{7}$ & 87 & $(166)$ \\
GHS_indef/c-68 & 0.01 & 1.28 & 2.32 & $2.7 \times 10^{7}$ & 37 & $(43)$ \\
GHS_indef/c-69 & 0.01 & 0.32 & 2.35 & $5.4 \times 10^{7}$ & 67 & $(73)$ \\
GHS_indef/c-70 & 0.01 & 0.32 & 2.33 & $6.0 \times 10^{7}$ & 71 & $(72)$ \\
GHS_indef/c-71 & 0.01 & 0.02 & 2.17 & $7.9 \times 10^{7}$ & 78 & $(88)$ \\
GHS_indef/c-72 & 0.01 & 0.32 & 2.39 & $6.3 \times 10^{7}$ & 67 & $(78)$ \\
Schenk_IBMNA/c-big & 0.01 & 0.64 & 2.63 & $3.8 \times 10^{8}$ & 109 & $(268)$ \\
\hline
\end{tabular}

For these interior-point problems, we found it was beneficial to regularize by using non-zero initial shifts: if we set $\alpha_{i n}(1: 2)=0.0$, in our tests the factorization did not break down on an $A$-node (so that $\left.\alpha_{\text {out }}(1)=0.0\right)$ but it was necessary to significantly increase the $C$-shift, leading to a much poorer quality preconditioner. This is illustrated in Table 6.10.

Table 6.10: GMRES(100) convergence results for Test Set 2 with lsize $=r$ size $=10$ and $\alpha_{i n}(1: 2)=0.0$. - denotes failure to converge within 1000 iterations.

\begin{tabular}{lrrrrr}
\hline Identifier & $\alpha_{\text {out }}(1)$ & $\alpha_{\text {out }}(2)$ & fill $_{I C}$ & efficiency & iters \\
\hline GHS_indef/c-55 & 0.0 & 16.4 & 2.06 & $1.3 \times 10^{8}$ & 281 \\
GHS_indef/c-59 & 0.0 & 32.8 & 2.17 & $2.2 \times 10^{8}$ & 382 \\
GHS_indef/c-63 & 0.0 & 32.8 & 2.30 & $2.1 \times 10^{8}$ & 383 \\
GHS_indef/c-68 & 0.0 & 131 & 2.34 & $2.9 \times 10^{8}$ & 398 \\
GHS_indef/c-69 & 0.0 & 65.5 & 2.39 & $2.4 \times 10^{8}$ & 288 \\
GHS_indef/c-70 & 0.0 & 32.8 & 2.31 & $3.2 \times 10^{8}$ & 379 \\
GHS_indef/c-71 & 0.0 & 32.8 & 2.16 & $3.7 \times 10^{8}$ & 362 \\
GHS_indef/c-72 & 0.0 & 65.5 & 2.39 & $4.5 \times 10^{8}$ & 471 \\
Schenk_IBMNA/c-big & 0.0 & 262 & - & - & - \\
\hline
\end{tabular}

To illustrate the effects of using larger lsize and rsize values, in Table 6.11 we present results for $l$ size $=r$ size $=30$. If we compare these with Table $6.9($ lsize $=r$ size $=10)$, we see that the fill increases (but is still much less than for a complete factorization) while the value of the $C$-shift is reduced. This leads to a reduction in the iteration count (by around 50 per cent for many of the problems) and an improvement in the efficiency for most cases.

\subsection{Comparisons with SYM-ILDL}

It is of interest to consider how the performance of our signed incomplete factorization preconditioner compares with that of an incomplete indefinite factorization that incorporates pivoting. The package we use for comparison is SYM-ILDL by Greif and Liu. As discussed in Section 1, this is based on the earlier work by $\mathrm{Li}$ and Saad [37] and performs an incomplete factorization of sparse symmetric indefinite matrices with Bunch-Kaufman pivoting [10] used for numerical stability (and to prevent breakdown). Thus it computes an incomplete factorization of the form $L D L^{T}$, where $D$ is block diagonal, with blocks of order 1 and 2 , corresponding to $1 \times 1$ and $2 \times 2$ pivots. The matrix is preordered using AMD or RCM and optionally 
Table 6.11: GMRES(100) convergence results for Test Set 2 with lsize $=$ rsize $=30$ and $\alpha_{i n}(1: 2)=0.01$. Results are also given for the code SYM-ILDL run with $f i l l=12.0$ and $t o l=0.003$. - denotes failure to converge within 1000 iterations.

\begin{tabular}{llcccr|rrr}
\hline \multicolumn{1}{c}{ Identifier } & \multicolumn{4}{c|}{ Signed IC } & \multicolumn{3}{c}{ SYM-ILDL } \\
& $\alpha_{\text {out }}(1)$ & $\alpha_{\text {out }}(2)$ & fill $I C$ & efficiency & iters & fill $_{I C}$ & efficiency & iters \\
\hline GHS_indef/c-55 & 0.01 & 0.08 & 3.37 & $3.0 \times 10^{7}$ & 41 & 4.35 & $7.4 \times 10^{7}$ & 78 \\
GHS_indef/c-59 & $2.5 \times 10^{-3}$ & 0.01 & 3.67 & $3.9 \times 10^{7}$ & 41 & 4.33 & $1.1 \times 10^{8}$ & 97 \\
GHS_indef/c-63 & 0.01 & 0.02 & 3.79 & $4.3 \times 10^{7}$ & 47 & 4.46 & $6.5 \times 10^{7}$ & 61 \\
GHS_indef/c-68 & 0.01 & 0.01 & 4.06 & $1.8 \times 10^{7}$ & 14 & 4.31 & $8.3 \times 10^{7}$ & 61 \\
GHS_indef/c-69 & $2.5 \times 10^{-3}$ & 0.01 & 4.00 & $4.1 \times 10^{7}$ & 30 & 3.81 & $5.5 \times 10^{7}$ & 42 \\
GHS_indef/c-70 & 0.01 & 0.01 & 3.88 & $6.3 \times 10^{7}$ & 45 & 3.61 & $2.9 \times 10^{7}$ & 22 \\
GHS_indef/c-71 & 0.01 & 0.01 & 3.51 & $8.7 \times 10^{7}$ & 53 & 4.03 & $7.0 \times 10^{7}$ & 37 \\
GHS_indef/c-72 & 0.01 & 0.01 & 3.91 & $5.7 \times 10^{7}$ & 37 & 4.02 & $9.7 \times 10^{7}$ & 61 \\
Schenk_IBMNA/c-big & 0.01 & 0.01 & 4.44 & $3.3 \times 10^{8}$ & 56 & 4.47 & - & - \\
\hline
\end{tabular}

prescaled to be equilibrated in the maximum norm. The input parameters that can be set by the user to control the number of entries within $L$ are fill and tol. Each column of the computed incomplete factor $L$ is guaranteed to have fewer than $\mathrm{fill} \cdot n e(K) / n$ entries, where $n e(K)$ is the number of entries in $K$ (upper and lower triangular parts). It has default value 1.0. The parameter tol controls the aggressiveness of the dropping of small entries. In each column $k$ of $L$, entries that are less than $t o l \cdot\left\|L_{k+1: n, k}\right\|_{1}$ in magnitude are discarded. The default setting for tol is 0.001 .

Table 6.11 includes results for SYM-ILDL for the Test Set 2 problems run with the settings fill $=12.0$ and $t o l=0.003$. With these choices, the level of fill is similar to that for the signed $I C$ factorization. We see that, in some cases (in particular, c-70), SYM-ILDL produces a higher quality preconditioner but, for other problems (including c-63 and c-68) the preconditioner computed by our signed $I C$ factorization is better.

We next consider Test Set $1(C=0)$. Results are given in Table 6.12. The input parameter fill for SYM-ILDL is chosen for each problem to give good performance with a level of fill in the factor that is generally similar to that for the signed $I C$ factorization; the drop tolerance $t o l=0.001$ is used. Problems GHS_indef/cont-201 and GHS_indef/cont-300 are omitted as SYM-ILDL did not converge. The signed $I C$ results are reproduced from Table 6.3. Again, we see that the signed $I C$ approach can outperform SYM-ILDL but for some problems (such as GHS_indef/brainpc2), the latter produces a higher quality preconditioner. We are not able to predict for which problems which approach will give the better results.

\section{Concluding remarks}

In this paper, we have looked at extending the robust limited-memory incomplete Cholesky factorization algorithm of $[56,57]$ to sparse symmetric indefinite systems in saddle-point form. By using two diagonal shifts to prevent breakdown, we are able to compute a signed incomplete Cholesky factorization of the form $L D L^{T}$, where the diagonal matrix $D$ has entries \pm 1 . Some new theoretical results have been given and numerical results presented to illustrate the effectiveness of the approach. The effects of different orderings and scalings on the preconditioner have also been investigated. As in the positive-definite case, we have shown that the use of intermediate memory can improve the quality of the preconditioner. Furthermore, the use of regularization parameters (that is, non-zero initial diagonal shifts) for the blocks $A$ and $-C$ has been found to substantially improve performance for interior-point optimization matrices. We have developed a new software package HSL_MI30 that implements our signed $I C$ factorization algorithm; this Fortran package (which also offers a MATLAB interface) is part of the HSL mathematical software library [34]. 
Table 6.12: GMRES(100) convergence results for Test Set 1. The settings for the signed IC code are as for Table 6.3.

\begin{tabular}{lrrr|rrrr}
\hline \multicolumn{1}{c}{ Identifier } & \multicolumn{3}{c|}{ Signed IC } & \multicolumn{4}{c}{ SYM-ILDL } \\
& fill IC $_{I C}$ & efficiency & iters & fill & fill $I C$ & efficiency & iters \\
\hline GHS_indef/aug3dcqp & 1.35 & $1.05 \times 10^{5}$ & 1 & 1.0 & 1.61 & $1.2 \times 10^{5}$ & 1 \\
GHS_indef/boyd1 & 0.82 & $3.76 \times 10^{6}$ & 7 & 1.0 & 0.86 & $1.3 \times 10^{7}$ & 23 \\
GHS_indef/brainpc2 & 1.28 & $1.64 \times 10^{7}$ & 132 & 1.0 & 0.72 & $1.5 \times 10^{6}$ & 22 \\
GHS_indef/d_pretok & 1.96 & $4.85 \times 10^{7}$ & 28 & 1.0 & 1.96 & $2.7 \times 10^{8}$ & 156 \\
\hline GHS_indef/darcy003 & 5.44 & $2.80 \times 10^{8}$ & 44 & 10.0 & 4.84 & $2.3 \times 10^{8}$ & 40 \\
GHS_indef/mario001 & 5.37 & $9.86 \times 10^{6}$ & 16 & 4.0 & 4.25 & $1.2 \times 10^{7}$ & 24 \\
GHS_indef/ncvxqp9 & 3.98 & $2.51 \times 10^{5}$ & 2 & 3.0 & 3.64 & $7.0 \times 10^{4}$ & 61 \\
GHS_indef/olesnik0 & 4.43 & $4.82 \times 10^{7}$ & 27 & 4.0 & 4.81 & $6.6 \times 10^{7}$ & 34 \\
GHS_indef/qpband & 1.17 & $3.50 \times 10^{4}$ & 1 & 0.5 & 1.67 & $3.5 \times 10^{4}$ & 1 \\
GHS_indef/sit100 & 3.61 & $1.72 \times 10^{6}$ & 14 & 5.0 & 3.24 & $6.2 \times 10^{6}$ & 56 \\
\hline GHS_indef/stokes64 & 2.73 & $3.19 \times 10^{7}$ & 157 & 2.0 & 3.73 & $1.1 \times 10^{8}$ & 379 \\
GHS_indef/stokes128 & 2.73 & $4.35 \times 10^{8}$ & 539 & 4.0 & 5.86 & $1.3 \times 10^{9}$ & 740 \\
GHS_indef/tuma1 & 5.79 & $5.86 \times 10^{6}$ & 20 & 15.0 & 4.91 & $3.2 \times 10^{6}$ & 13 \\
GHS_indef/tuma2 & 6.55 & $3.36 \times 10^{6}$ & 18 & 15.0 & 4.66 & $1.4 \times 10^{6}$ & 11 \\
GHS_indef/turon_m & 4.36 & $2.23 \times 10^{8}$ & 56 & 6.0 & 5.58 & $3.5 \times 10^{8}$ & 67 \\
\hline
\end{tabular}

We have presented some numerical results for the recent SYM-ILDL package, which uses BunchKaufman pivoting to avoid breakdown. In the case of a direct solver, it has been reported [54] that a signed Cholesky factorization code in general performs less well than a carefully engineered indefinite code that incorporates threshold partial pivoting. However, our results show that our limited-memory signed $I C$ approach can be competitive with SYM-ILDL. It is now our intention to develop an incomplete indefinite factorization code that incorporates pivoting and also uses intermediate memory.

\section{Acknowledgements}

We are grateful to Chen Greif and Paul Liu for discussions around their code SYM-ILDL and for advice on computing the numerical results for SYM-ILDL that are reported in Sections 6.3 and 6.4. Thanks also to Chen for carefully reading and commenting on a draft of this paper. The version of SYM-ILDL used was downloaded from https://github.com/inutard/matrix-factor on 10 December 2013. All results were performed using the HSL implementations of SYMMBK and GMRES (available from http: //www.hsl.rl.ac.uk/catalogue/).

\section{References}

[1] G. Al-Jeiroudi, J. Gondzio, and J. Hall. Preconditioning indefinite systems in interior point methods for large scale linear optimisation. Optimization Methods and Software, 23(3):345-363, 2008.

[2] A. Altman and J. Gondzio. Regularized symmetric indefinite systems in interior point methods for linear and quadratic optimization. Optimization Methods and Software, 11/12(1-4):275-302, 1999. Interior point methods.

[3] P. R. Amestoy, T. A. Davis, and I. S. Duff. Algorithm 837: AMD, an approximate minimum degree ordering algorithm. ACM Transactions on Mathematical Software, 30:381-388, 2004.

[4] M. Benzi, G.H. Golub, and J. Liesen. Numerical solution of saddle point problems. Acta Numerica, 14:1-137, 2005.

[5] M. Benzi and A. Wathen. Some preconditioning techniques for saddle point problems. In Model Order Reduction: Theory, Research Aspects and Applications, volume 13 of Mathematics in Industry, pages 195-211. Springer, 2008. 
[6] L. Bergamaschi, J. Gondzio, and G. Zilli. Preconditioning indefinite systems in interior point methods for optimization. Computational Optimization and Applications, 28(2):149-171, 2004.

[7] A. Berman and R. J. Plemmons. Nonnegative Matrices in the Mathematical Sciences. Academic Press, New York, 1979.

[8] S. Bonettini and V. Ruggiero. Some iterative methods for the solution of a symmetric indefinite KKT system. Computational Optimization and Applications, 38(1):3-25, 2007.

[9] R. Bridson. An ordering method for the direct solution of saddle-point matrices, 2007. Unpublished preprint available from http://www.cs.ubc.ca/ rbridson/kktdirect/.

[10] J. R. Bunch and L. Kaufman. Some stable methods for calculating inertia and solving symmetric linear systems. Mathematics of Computation, 31:162-179, 1977.

[11] X. Chen, K.-K. Phoon, and K.-C. Toh. Performance of zero-level fill-in preconditioning techniques for iterative solutions with geotechnical applications. International Journal of Geomechanics, 12:596-605, 2012.

[12] E. Chow and Y. Saad. Experimental study of $I L U$ preconditioners for indefinite matrices. J. of Computational and Applied Mathematics, 86(2):387-414, 1997.

[13] E. H. Cuthill and J. McKee. Reducing the bandwidth of sparse symmetric matrices. In Proceedings $24^{\text {th }}$ National Conference of the ACM, pages 157-172. ACM Press, 1969.

[14] T. A. Davis and Y. Hu. The University of Florida sparse matrix collection. ACM Transactions on Mathematical Software, 38(1), 2011.

[15] A. C. de Niet and F. W. Wubs. Numerically stable $L D L^{T}$-factorization of F-type saddle point matrices. $I M A$ Journal of Numerical Analysis, 29:208-234, 2009.

[16] I. S. Duff. MA57- a new code for the solution of sparse symmetric definite and indefinite systems. ACM Transactions on Mathematical Software, 30:118-154, 2004.

[17] I. S. Duff and J. Koster. On algorithms for permuting large entries to the diagonal of a sparse matrix. SIAM J. on Matrix Analysis and Applications, 22:973-996, 2001.

[18] I. S. Duff and G. A. Meurant. The effect of ordering on preconditioned conjugate gradients. BIT Numerical Mathematics, 29:635-657, 1989.

[19] I. S. Duff and S. Pralet. Strategies for scaling and pivoting for sparse symmetric indefinite problems. SIAM J. on Matrix Analysis and Applications, 27:313-340, 2005.

[20] I. S. Duff and J. A. Scott. Towards an automatic ordering for a symmetric sparse direct solver. Technical Report RAL-TR-2006-001, Rutherford Appleton Laboratory, 2005.

[21] V. Eijkhout. Analysis of parallel incomplete point factorizations. Linear Algebra and its Applications, 154/156:723-740, 1991.

[22] H. C. Elman and G. H. Golub. Inexact and preconditioned Uzawa algorithms for saddle point problems. SIAM J. on Numerical Analysis, 31:1645-1661, 1994.

[23] R. Fourer and S. Mehrotra. Solving symmetric indefinite systems in an interior-point method for linear programming. Math. Programming, 62(1, Ser. B):15-39, 1993.

[24] P. E. Gill, M. A. Saunders, and J. R. Shinnerl. On the stability of Cholesky factorization for symmetric quasidefinite systems. SIAM J. Matrix Anal. Appl., 17(1):35-46, 1996.

[25] G. H. Golub and C. F. Van Loan. Unsymmetric positive definite linear systems. Linear Algebra and its Applications, 28:85-97, 1979.

[26] J. Gondzio. HOPDM (version 2.12) - a fast LP solver based on a primal-dual interior point method. European Journal of Operational Research, 85(1):221-225, 1995.

[27] C. Greif. Preconditioners for linear systems arising from interior-point methods. Presentation at the International Conference On Preconditioning Techniques for Scientific and Industrial Applications, The University of Oxford, 2013.

[28] C. Greif, S. He, and P. Liu. SYM-ILDL: C++ package for incomplete factorizations of symmetric indefinite matrices. https://github.com/inutard/matrix-factor, 2013. 
[29] M. Hagemann and O. Schenk. Weighted matchings for preconditioning symmetric indefinite linear systems. SIAM J. on Scientific Computing, 28(2):403-420, 2006.

[30] J. D. Hogg and J. A. Scott. The effects of scalings on the performance of a sparse symmetric indefinite solver. Technical Report RAL-TR-2008-007, Rutherford Appleton Laboratory, 2008.

[31] J. D. Hogg and J. A. Scott. An indefinite sparse direct solver for large problems on multicore machines. Technical Report RAL-TR-2010-011, Rutherford Appleton Laboratory, 2010.

[32] J. D. Hogg and J. A. Scott. HSL_MA97: a bit-compatible multifrontal code for sparse symmetric systems. Technical Report RAL-TR-2011-024, Rutherford Appleton Laboratory, 2011.

[33] J. D. Hogg and J. A. Scott. Pivoting strategies for tough sparse indefinite systems. ACM Transactions on Mathematical Software, 40, 2013. Article 4, 19 pages.

[34] HSL. A collection of Fortran codes for large-scale scientific computation, 2013. http://www.hsl.rl.ac.uk.

[35] I. E. Kaporin. High quality preconditioning of a general symmetric positive definite matrix based on its $U^{T} U+U^{T} R+R^{T} U$ decomposition. Numerical Linear Algebra with Applications, 5:483-509, 1998.

[36] D. S. Kershaw. The incomplete Cholesky-conjugate gradient method for the iterative solution of systems of linear equations. J. of Computational Physics, 26:43-65, 1978.

[37] N. Li and Y. Saad. Crout versions of ILU factorization with pivoting for sparse symmetric matrices. Electronic Transactions on Numerical Analysis, 20:75-85, 2005.

[38] N. Li, Y. Saad, and E. Chow. Crout versions of ILU for general sparse matrices. SIAM J. on Scientific Computing, 25(2):716-728, 2003.

[39] S. X. Li and J. W. Demmel. Making parallel gaussian elimination scalable by static pivoting. In Proceedings of SuperComputing'98, November 7-13, 1998, Orlando, FL, pages 519-523, 1998.

[40] C.-J. Lin and J. J. Moré. Incomplete Cholesky factorizations with limited memory. SIAM J. on Scientific Computing, 21(1):24-45, 1999.

[41] T. A. Manteuffel. An incomplete factorization technique for positive definite linear systems. Mathematics of Computation, 34:473-497, 1980.

[42] O. Marques. Skypack user's guide. Technical Report, National Energy Research Scientific Computing Center (NERSC), Lawrence Berkeley National Laboratory, 2009.

[43] J. A. Meijerink and H. A. van der Vorst. An iterative solution method for linear systems of which the coefficient matrix is a symmetric $M$-matrix. Mathematics of Computation, 31(137):148-162, 1977.

[44] A. Messaoudi. On the stability of the incomplete $L U$-factorizations and characterizations of $H$-matrices. Numerische Mathematik, 69(3):321-331, 1995.

[45] D. Orban. Limited-memory LDLT factorization of symmetric quasi-definite matrices. GERAD Technical Report G-2013-87, 2013.

[46] C. C. Paige and M. A. Saunders. Solution of sparse indefinite systems of linear equations. SIAM J. on Numerical Analysis, 12(4):617-629, 1975.

[47] J. K. Reid and J. A. Scott. Ordering symmetric sparse matrices for small profile and wavefront. International J. of Numerical Methods in Engineering, 45:1737-1755, 1999.

[48] W. Ren and J. Zhao. Iterative methods with preconditioners for indefinite systems. J. of Computational Mathematics, 17:89-96, 1999.

[49] M. Rozložník, A. Smoktunowicz, and F. Okulicka-Dłużewska. Indefinite orthogonalization with rounding errors, submitted for publication. 2013.

[50] D. Ruiz. A scaling algorithm to equilibrate both rows and columns norms in matrices. Technical Report RAL-TR-2001-034, Rutherford Appleton Laboratory, Chilton, Oxfordshire, England, 2001.

[51] D. Ruiz and B. Uçar. A symmetry preserving algorithm for matrix scaling. Technical Report INRIA RR-7552, INRIA, Grenoble, France, 2011.

[52] M. A. Saunders and J. A. Tomlin. Solving regularized linear porograms using barrier methods and KKT systems. Technical Report SOL-96-4, SOL, Department of Operations Research, Stanford University, 1996. 
[53] O. Schenk and K. Gärtner. On fast factorization pivoting methods for symmetric indefinite systems. Electronic Transactions on Numerical Analysis, 23:158-179, 2006.

[54] J. A. Scott. A note on a simple constrained ordering for saddle-point systems. Technical Report RAL-TR2009-007, Rutherford Appleton Laboratory, 2009.

[55] J. A. Scott and M. Tůma. The importance of structure in incomplete factorization preconditioners. BIT Numerical Mathematics, 51:385-404, 2011.

[56] J. A. Scott and M. Tůma. HSL_MI28: an efficient and robust limited memory incomplete Cholesky factorization code. ACM Transactions on Mathematical Software, 2014. To appear.

[57] J. A. Scott and M. Tůma. On positive semidefinite modification schemes for incomplete Cholesky factorization. SIAM J. on Scientific Computing, 2014. To appear.

[58] S. W. Sloan. An algorithm for profile and wavefront reduction of sparse matrices. International J. of Numerical Methods in Engineering, 23:239-251, 1986.

[59] S. W. Sloan. A Fortran program for profile and wavefront reduction. International J. of Numerical Methods in Engineering, 28:2651-2679, 1989.

[60] M. Tůma. A note on the LDLT decomposition of matrices from saddle-point problems. SIAM J. on Matrix Analysis and Applications, 23(4):903-915, 2002.

[61] M. ur Rehman, C. Vuik, and G. Segal. A comparison of preconditioners for incompressible navier-stokes solvers. International J. for Numerical Methods in Fluids, 57:1731-1751, 2008.

[62] H. A. van der Vorst. Iterative Krylov Methods for Large Linear Systems. Cambridge Monographs on Applied and Computational Mathematics, Cambridge University Press, Cambridge, UK, 2003.

[63] R. J. Vanderbei. Symmetric quasidefinite matrices. SIAM J. on Optimization, 5(1):100-113, 1995.

[64] R. J. Vanderbei. LOQO user's manual-version 3.10. Optimization Methods and Software, 11/12(1-4):485-514, 1999.

[65] R. J. Vanderbei and D. F. Shanno. An interior-point algorithm for nonconvex nonlinear programming. Computational Optimization and Applications, 13(1-3):231-252, 1999.

[66] R. S. Varga, E. B. Saff, and V. Mehrmann. Incomplete factorizations of matrices and connections with H-matrices. SIAM J. on Numerical Analysis, 17:787-793, 1980.

[67] J. Zhao. The generalized Cholesky factorization method for saddle-point problems. Applied Mathematics and Computation, 92:49-58, 1998. 